\title{
Enhancing Customer Relationship Management by Integrating Customer and Product Values based on Clustering and Indexing Techniques
}

\author{
V.Jayaraj \\ Associate Professor \\ Bharathidasan University \\ Tamilnadu, India
}

\author{
M.Rajakumar \\ Research Scholar \\ Periyar University \\ Tamilnadu, India
}

\author{
J.Lavanya \\ Research Scholar \\ Bharathidasan University \\ Tamilnadu, India
}

\author{
J.Jegathesh Amalraj \\ Research Scholar \\ Bharathidasan University \\ Tamilnadu, India
}

\begin{abstract}
Customer relationship management is not only pure business, but also indicates strong personal bonding within people. Development of this type of bonding drives the business to new levels of success. Once this personal and emotional linkage is built, it is very easy for any organization to identify the actual needs of the customer and to help and serve them in a better way. It is a belief that if more sophisticated strategies are involved in implementing the customer relationship management, the business becomes stronger and fruitful.The main objective of this paper is to provide an optimal solution which optimizes the process of Customer Relationship Management. The process is primarily executed by means of the Clustering technique. When a new customer arrives, that customer is classified into any one of the existing Clusters which automatically provides us the properties of the customer. This paper presents two phases of Clustering method which is not only the clusters on the base of an only attribute, but performs a secondary clustering operation for improved and precise results. Additionally all the Clustered information is indexed in such a manner that Clustering come to be faster.
\end{abstract}

Keywords: Customer Relationship Management, Customer Value, Clustering, Classification, Two Phase Clustering, Indexing

\section{INTRODUCTION}

Customer relationship management incorporates the information acquisition, information storage, and decision support functions to provide customized customer service. It enables customer representatives to analyse data and address customer needs in order to promote greater customer satisfaction and retention. It helps organizations to interact with their customers through a variety of means including phone, web, e-mail, and salesperson. Customer representatives can access data on customer profile, product, logistics and the like to analyse problems and provide online and rapid response to customer queries.

Customer Relationship management is the robust and the greatest effective approach in keeping and producing relationships with customers. This personal and emotional association considered to be very helpful for any organization to find the tangible needs of customer and help them to serve in a better way. It is an acceptance that more the refined approaches elaborate in implementing the customer relationship management, the more robust and productive is the business. Development of this type of bonding impulses the business in to a new level of success.
CRM has been firstly performed manually by an employee of the organization. Because of the increase in online transactions, it becomes improved and effective to automate this process [8] [9].

An appropriate mathematical model cannot be suggested for this strategy, since this process becomes changeable, as it embroils human being as a key element. Once this type of consequence is met, a constant learning algorithm is a key to success [4]. Our paper proposes such an algorithm incorporating the elements of Data Mining.

The main objective of this paper is to provide an optimal solution which optimizes the process of Customer Relationship Management. The process is primarily executed by means of the Clustering technique .When a new customer arrives, that customer is classified into any one of the existing Clusters which automatically provides us the properties of the customer. This paper presents two phases Clustering method which not only the clusters on the base of a single attribute, but performs a secondary clustering operation for improved and precise results. Additionally all the Clustered information is indexed in such a manner that Clustering come to be faster.

\section{METHODOLOGY}

Data mining, which is also called as KDD (Knowledge Discovery in Database).It is the process of extracting unacquainted, potential and valuable information and knowledge from bountiful, inadequate, noisy, fuzzy and stochastic real data. It is a method to pick up the information and knowledge which cannot be revealed straight but with possible value from a greater part of data. The suggesting method uses the process of Clustering, Classification and Neural Network techniques for the mining data.

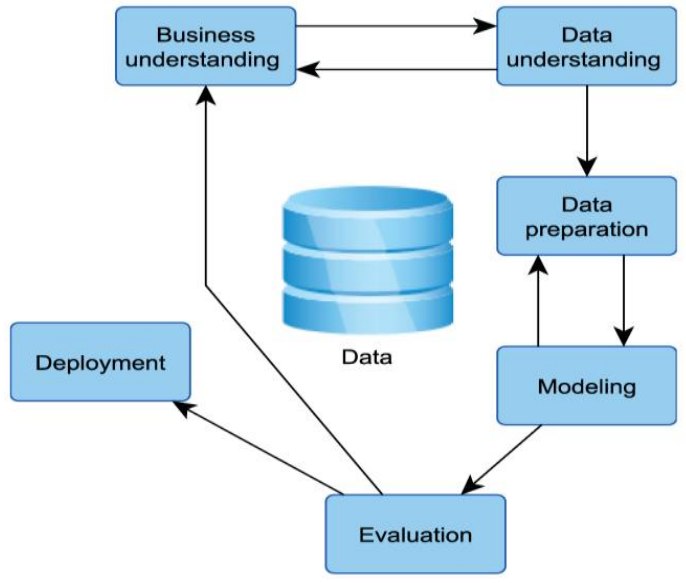

Fig. 1: Data Mining Process 


\subsection{Back Propagation Neural Network}

An artificial neural network (ANN), often just called a "neural network" ( $\mathrm{NN})$, is a mathematical model or computational model based on biological neural networks, in other words, is a simulation of biological neural system. It consists of a connected group of artificial neurons and processes information using a connectionist approach to computation.

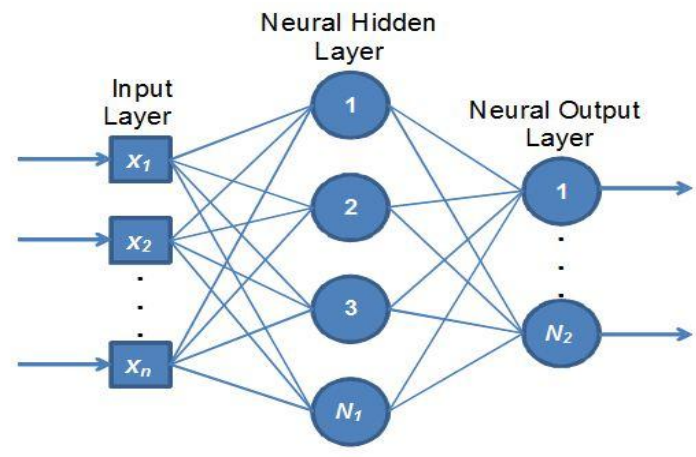

Fig. 2: Structure of an Artificial Neural Network

An ANN is an adaptive system that changes its structure based on external or internal information that flows through the network during the learning phase.

An ANN is classically defined by three categories of parameters:

1. The interconnection pattern between different layers of neurons

2. The learning process for updating the weights of the interconnections

3. The activation function that converts a neuron's weighted input to its output activation.

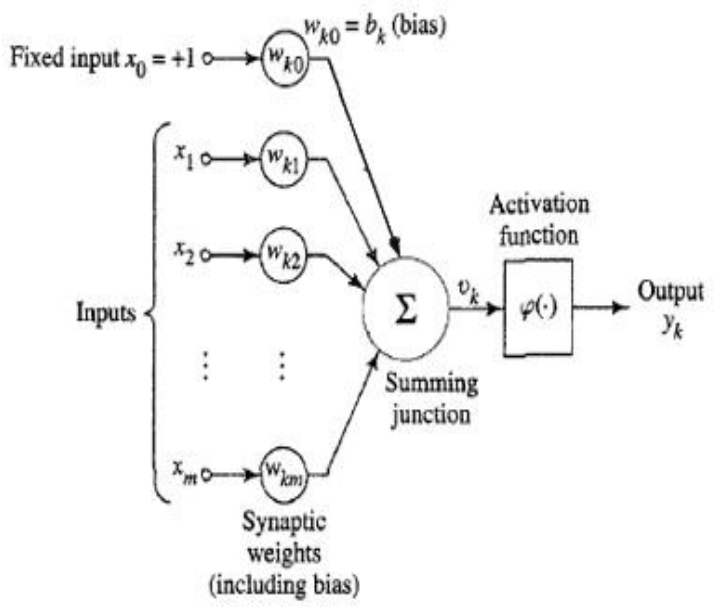

Fig. 3: Mathematical Model of a Neural Network

\subsection{Clustering and Classification process}

Data clustering is a technique in which we make cluster of objects that are somehow similar in characteristics. The measure for checking the similarity is implementation reliant. Data Clustering is a method in which, the information that is logically similar is physically stored together. Data classification is the classification of data for its most effective and competent use. In a basic approach to storing computed data, data can be classified according to its critical value or how often it desires to be accessed, with the greatest critical or often-used data stored on the fastest media while other data can be stored on slower (and less expensive) media. By applying these techniques in CRM, the process of customer analysis come to be further accurate. [2], [3], [7]

\section{THE CUSTOMER RELATIONSHIP MANAGEMENT PROCESS}

The CRM process is carried out in two different phases. The first phase deals with processing of the data for the first time and further processing are carried out affording to the rules stated in the second phase.

The first phase involves gathering the required data from the user and performing the operation of Clustering on the obtained data. This results in producing clusters of similar types of data, as described in [1]. Further refine in this process done by performing clustering operation on the already clustered data, which provides us a further degree of refinement [10]. After the successful completion of this process, the acquired clusters are analyzed for common information. This information is recorded along with the common properties used for Clustering process. This forms the basis for Indexing.

When a new customer arrives, he/she is classified into any one of the existing clusters. The property of the customer is said to correspond to the properties of the cluster in which they are classified into.

The second phase deals with providing the user a faster performance during the clustering process. After a certain period of time, the data existing in the data store increases and henceforward a need for re-clustering arises. This process is usually tedious, since the entire first phase is carried out with a larger amount of data.

But in this proposed paper, the data existing in the clusters are indexed, then obtaining of properties from an indexed format is frequently faster than the normal database, so a large amount of time will be saved. The new data entries in the system have to be analyzed and stored in the index. This saves a significant amount of time and processing power.

Both, the clustering and the classification phases are performed using the artificial neural networks.

\subsection{Phase I}

The historical purchase data [6] that is existing in the organization under study is taken as the training data for the neural networks. [4] Each property of the customer is fed into a perceptron. The perceptron are already providing with weight-age values that correspond to the property represented by it.
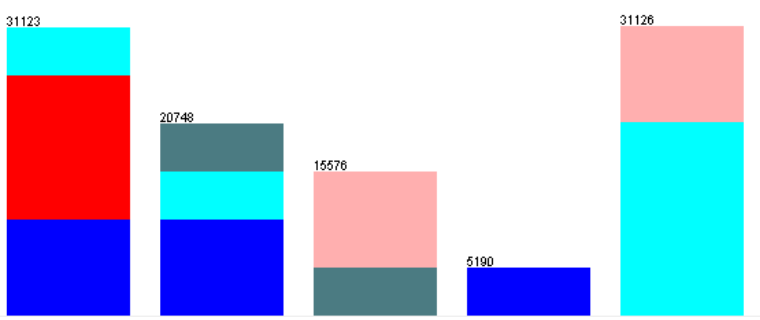

Fig. 4: Histogram representing the salary values of customers (Output from Weka 3.0) 
The second hidden layer consists of one perceptron that inputs all the values provided by the first layer. These properties all are processed and ending value called the Customer Value [5] is acquired from the system.
A similar process is conceded out for all the products that are being sold by the organization and a product value is obtained for each product.

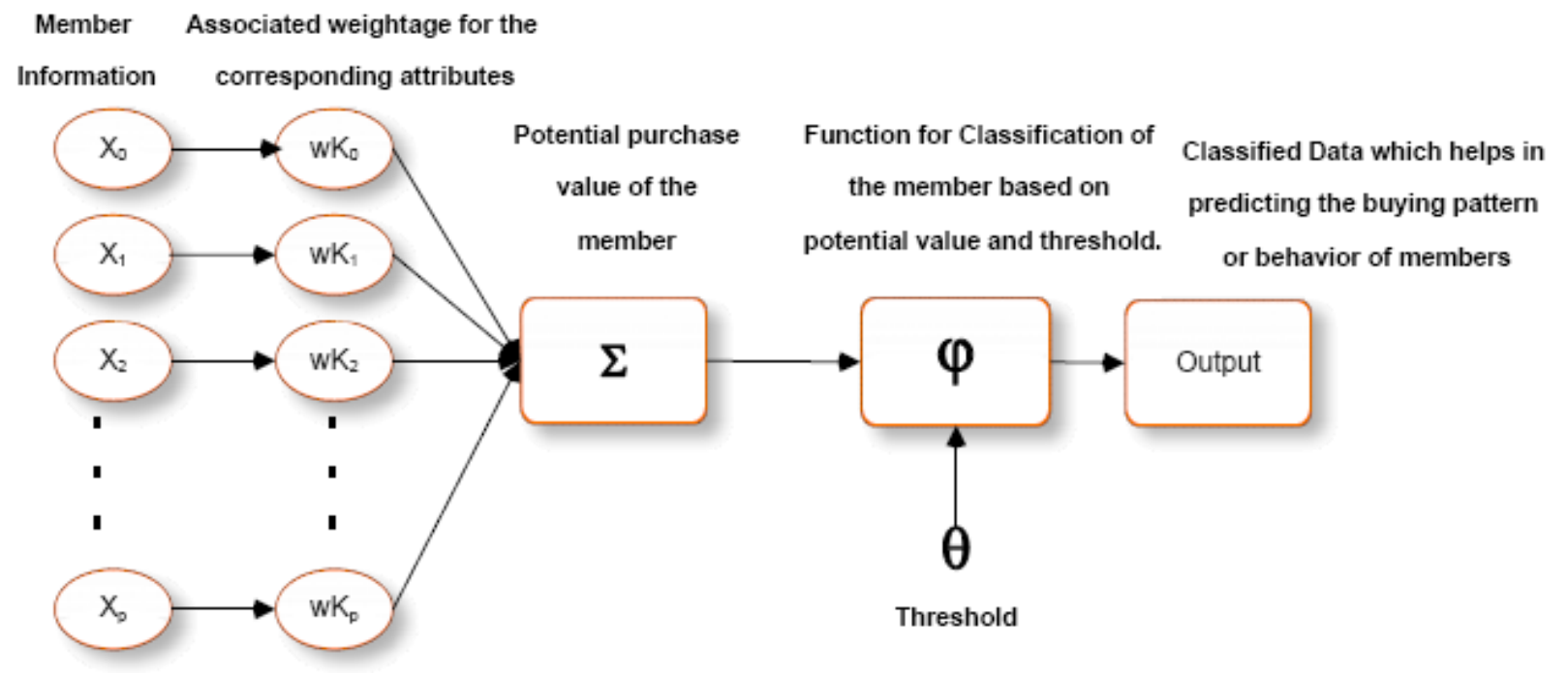

Fig. 5: User Profile Evaluation (Process of Clustering)

The minimum and maximum customer values are obtained from the result and a median value is calculated. This value acts as the base for Clustering. A boundary is defined by the user, with the obtained median value taken as the centroid. All the customer values that come under this boundary are added to the cluster.

A second phase Clustering is conceded out on each of these obtained clusters as defined in [10]. These clusters will correspond to a new property provided by the customer. This provides us with an additional division in each cluster or a second degree of refinement.

Each cluster is examined and similar properties are extracted out of each cluster and are considered as the properties belonging to the cluster. This property directly corresponds to the purchase capacity of the customer, or we can also say that these properties reflect the type of products that a customer most likely purchases. A similar process is carried out for the products cluster and product properties are obtained. All these data obtained are recorded in an indexed table. This table contains the customer value, the property values that were used in the clustering process and the cluster under which the current customer is present.

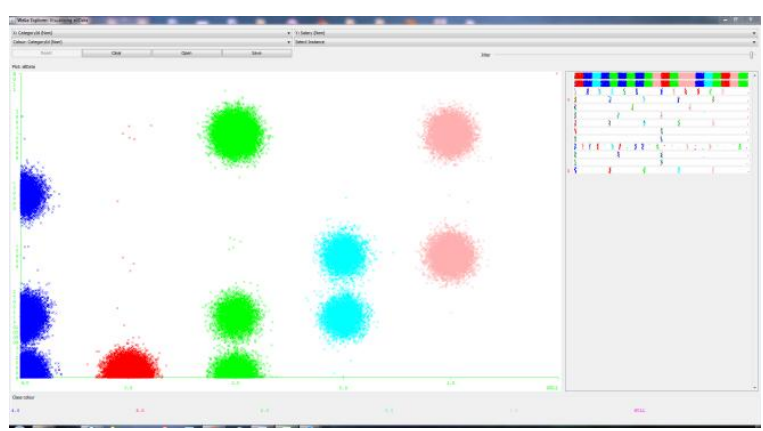

Fig. 6: Clusters corresponding to salary and purchase category (Output from Weka 3.0)

\section{Product Associated weightage for the \\ Information corresponding attributes}

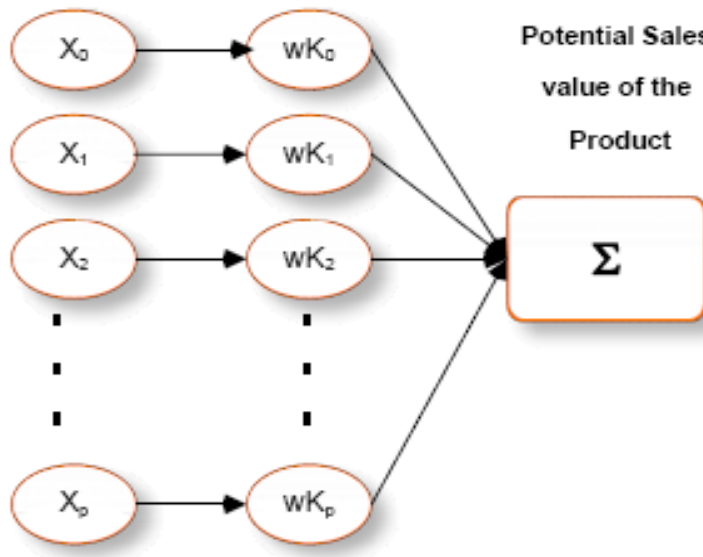

Function for Classification of the product based on potential

value and threshold.
Classified Data which helps in predicting the sales pattern of the product

Fig. 7: Process of Classification 
When a new customer data is provided to the system, the customer's properties are passed to the perceptron and they are passed to the first level hidden layer. This layer contains the final weight-age values that have been tuned in for the particular system. These weight-age values are processes with the equivalent properties and the results are passed to the second level hidden layer. The second level perceptron processes this cumulative information and the customer's value is obtained. The obtained customer value is compared with the existing clusters and it is classified onto a cluster that has the current customer value within its boundaries. This process is called Classification. [9]

The properties of the Cluster, under which the particular customer is classified, are taken as the properties corresponding to the customer. These properties are compared with the properties that have been generated from the product clusters. The two clusters are integrated to provide a solution to the user that contains all the products that the customer has the highest probability of buying.

\subsection{Phase II}

After a period of time, the database is prone to expand. When this happens, the user is compelled to carry out the clustering process again. This process keeps getting tedious every time this stage is reached, since every time a larger amount of data is to be handled by the user. This can be solved by using indexing. Instead of referring the entire database, the user can refer to the indexed values alone, which is faster. The new values are added to the table every time this process is carried out. Hence, processing is done only for the newly arrived data.

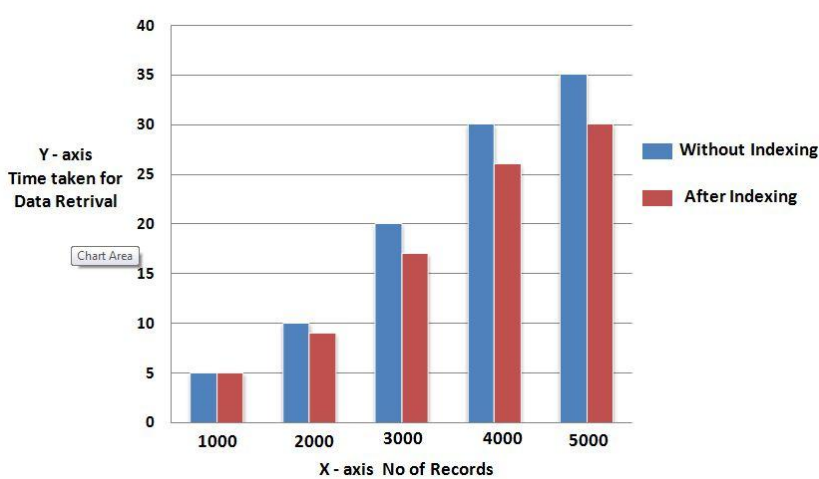

Fig. 8: Comparison Time Chart with and without Indexing

\section{CONCLUSION}

This proposed approach is the enhancement of the previous work [1]. Even though [1] gives us a general solution, it is still a tedious process every time the processing is conceded out. The phase I running time is found to increase periodically, hence it cannot provide an optimal solution for extended periods of time. Using the currently proposed method, the running time can be reduced to a large extent. Hence the usability of the system is found to increase. Since a two-way clustering is performed on the data, the currently proposed project provides a better solution.

The future work is to focus on the customer retention technique to enhance customer relationship via Data Mining techniques which leads to a good profit. The current process can be further extended by including the demographic and psychographic data about the customers. This type of segmentation leads to better analysis of customers and it might prove to be a valuable addition to the project that makes the results more accurate

\section{REFERENCES}

[1] Jayaraj V and J.Jegathesh Amalraj, Enhancing Customer Relationship Management Based on Customer and Product Value Estimation - A Neural Networks Approach, International Conference on Networks, Intelligence and Computing Technologies ICNICT'11, (2011).

[2] Wanghualin, Yuzhongdong, The Research of Customer Knowledge Management in CRM, International Conference on Intelligent Computation Technology and Automation (2010).

[3] Gao Jie, Customer Value Based On Data Mining, International Conference on Educational and Network Technology ICENT, (2010).

[4] Smita Nirkhi, Potential use of Artificial Neural Network in Data Mining, 978-1-4244-5586-7/10, IEEE, (2010).

[5] Xu Ling, Song Li, CRM - Customer Value Based On Data Mining, Third International Conference on Knowledge Discovery and Data Mining (2010).

[6] Gu Yu, Li Jiahui, Hierarchy analysis and optimization of customer value based on data mining technology, International Conference on Intelligent Computation Technology and Automation, (2010).

[7] Kun Wu, Feng-ying Liu, Application of Data Mining in Customer Relationship Management, 978-1-4244-53269/10, IEEE, (2010).

[8] Gao Hua, Customer Relationship Management Based on Data Mining Technique, 978-1-4244-8694-6/11, IEEE, (2011).

[9] Wanghualin, Data Mining and Its Applications in CRM, Second International Conference on Computer Research and Development, (2010).

[10] Namvar, Gholamian, KhakAbi, A Two Phase Clustering Method for Intelligent Customer Segmentation, 978-14244-5984-1, IEEE, (2011). 